\title{
Empowering Teachers with AI: Co-Designing a Learning Analytics Tool for Personalized Instruction in the Science Classroom
}

\author{
TANYA NAZARETSKY, Weizmann Institute of Science, Israel \\ CARMEL BAR, Weizmann Institute of Science, Israel \\ MICHAL WALTER, Weizmann Institute of Science, Israel \\ GIORA ALEXANDRON, Weizmann Institute of Science, Israel
}

\begin{abstract}
AI-based educational technology that is designed to support teachers in providing personalized instruction can enhance their ability to address the needs of individual students, hopefully leading to better learning gains. This paper presents results from a participatory research aimed at co-designing with science teachers a learning analytics tool that will assist them in implementing a personalized pedagogy in blended learning contexts. The development process included three stages. In the first, we interviewed a group of teachers to identify where and how personalized instruction may be integrated into their teaching practices. This yielded a clustering-based personalization strategy. Next, we designed a mock-up of an AI-based tool that supports this strategy and worked with another group of teachers to define an 'explainable learning analytics' scheme that explains each cluster in a way that is both pedagogically meaningful and can be generated automatically. Third, we developed an AI algorithm that supports this 'explainable clusters' pedagogy and conducted a controlled experiment that evaluated its contribution to teachers' ability to plan personalized learning sequences. The planned sequences were evaluated in a blinded fashion by an expert, and the results demonstrated that the experimental group teachers who received the clusters with the explanations - designed sequences that addressed the difficulties exhibited by different groups of students better than those designed by teachers who received the clusters without explanations. The main contribution of this study is twofold. First, it presents an effective personalization approach that fits blended learning in the science classroom, which combines a real-time clustering algorithm with an explainable-AI scheme that can automatically build pedagogically meaningful explanations from item-level meta-data ( $Q$ Matrix). Second, it demonstrates how such an end-to-end learning analytics solution can be built with teachers through a co-design process and highlights the types of knowledge that teachers add to system-provided analytics in order to apply them to their local context. As a practical contribution, this process informed the design of a new learning analytics tool that was integrated into a free online learning platform that is being used by more than 1000 science teachers.
\end{abstract}

Additional Key Words and Phrases: Personalized Instruction; Blended Learning; Teacher Dashboards; Participatory Design; Learning Analytics

ACM Reference Format:

Tanya Nazaretsky, Carmel Bar, Michal Walter, and Giora Alexandron. 2021. Empowering Teachers with AI: Co-Designing a Learning Analytics Tool for Personalized Instruction in the Science Classroom. In .... ACM, New York, NY, USA, 16 pages. https://doi.org/10.

1145/nnnnnnn.nnnnnnn

PRE-PRINT VERSION

Accepted to The 12th International Learning Analytics and Knowledge Conference (LAK'22).

Permission to make digital or hard copies of all or part of this work for personal or classroom use is granted without fee provided that copies are not made or distributed for profit or commercial advantage and that copies bear this notice and the full citation on the first page. Copyrights for components of this work owned by others than ACM must be honored. Abstracting with credit is permitted. To copy otherwise, or republish, to post on servers or to redistribute to lists, requires prior specific permission and/or a fee. Request permissions from permissions@acm.org.

(c) 2021 Association for Computing Machinery.

Manuscript submitted to ACM 


\section{INTRODUCTION}

Research has shown that personalized instruction - adapting various attributes of the learning process such as the pace and the content to the needs of the individual student - can significantly enhance learning outcomes [7], and that teachers are also aware of its potential [4, 32]. However, implementing personalized instruction in the K-12 classroom settings is acknowledged to be complex [41]. It requires constant monitoring of student-level factors that the instruction is personalized accordingly (most commonly, student knowledge, e.g., cognitive-based personalization, but affective state [29], student interest [40], and other dimensions may also be considered), finding available treatments that match individual needs, and means to orchestrate classroom activities in which students (or groups of students) follow different learning paths $[11,26,27]$.

Blending technology into the classroom, and in particular, Artificial Intelligence (AI) and Learning Analytics tools that provide actionable analytics that are based on rich process data, have the potential to support teachers in adopting personalized pedagogy $[6,19,21,31]$. Indeed, during the last decade, several tools (e.g., educational dashboards, teaching augmentation tools) were designed within this conceptual framework (see for example [3, 42]). But despite these efforts, teachers often do not adopt such tools either because they lack training in data-driven teaching practices and skills, or because the tools either do not fit class reality or are not suitable for the teachers' pedagogical preferences and habits $[15,35]$. In addition, there is also the issue of self-efficacy: to adopt educational technology, teachers should trust both the technology and their own ability to master it $[16,17]$.

Participatory or co-design processes that involve teachers throughout the design stages may be a useful approach for designing solutions that address teachers' needs and can gain their trust [14,28]. The rationale is that involving teachers, who are the end-users of the technology, working on authentic problems in real-life settings, and bringing together a multidisciplinary team in terms of skills and perspectives, would increase the likelihood that the design process will produce tools that teachers would perceive as useful and practical to their work [12, 22]. This is especially true for applied educational research such as ours, which aims at making an impact on the field - a challenge that is human-oriented not less than it is a technological one [38]. In this regard, the experience of teachers and instructors is of particular importance [41], and while learning analytics may be a relatively new domain, it is good to remember that making pedagogical decisions based on student data is definitely not a new component of the teaching practice [8].

In spite of these benefits, co-design processes are rarely followed in AI-based tool design, among other things, due to the difficulties in involving non-technical stakeholders in the design process [28]. Teachers usually 'speak' different language than the technical team and may lack advanced data literacy and computational skills, potentially limiting their understanding of how learning data can be used and what are the capabilities of AI-powered technologies [22, 23]. That said, the technical nature of learning analytics is indeed a challenge for participatory design with teachers, but not an absolute barrier [12].

Our research followed a participatory design process with science teachers to develop a learning analytics tool that will enhance their ability to define individualized learning sequences that are tailored to the needs of individual students. The study sought to answer the following three research questions:

- RQ1: In which ways do high-school science teachers believe that personalized instruction can be effectively integrated into their teaching practices?

- RQ2: What types of analytics can assist teachers in implementing the practices defined in RQ1 in their class?

- RQ3: Does providing teachers with the analytics defined in RQ2 increase their ability to adapt the instruction to the needs of learners with different types of difficulties? 
To answer these questions, we followed a co-design process that included three consecutive experiments with science teachers. In the first, a group of early-adopter teachers who frequently blend digital learning resources into their teaching was interviewed to understand where and how personalization can be integrated into their daily teaching practices. Based on the results, a personalization strategy was conceptualized and a mock-up of a learning analytics tool that supports it was developed. In the second experiment, another group of pilot teachers was presented with the personalization strategy and the mock-up in order to learn whether they find it valuable, and if so, what type of analytics they would like to receive through the tool, and how they would like these analytics to be explained in a pedagogically meaningful way. Then, we conducted a third, controlled experiment in which we examined whether providing these analytics to teachers actually enhances their ability to provide personalized learning sequences.

This research is part of our overarching goal of empowering teachers with AI-based learning analytics tools that are designed for blended environments for science teaching. Following the participatory study reported below, a new learning analytics tool that implements the conceptual design that was evaluated in this research was developed and integrated into PeTeL, a free online learning environment that is available to more than 1000 high school science teachers in Israel. With this tool, the learning platform would assist teachers in implementing more personalized instruction in which students receive learning sequences that are tailored to their current knowledge based on multi-dimensional, real-time analysis.

The rest of this paper is organized as follows. In Section 2, we present the high-level research design. Then, we present the methodology, analysis, and findings of each one of the three experiments conducted within this research. In Section 6, we present the design and implementation of the tool. Section 7 provides a joint discussion, followed by summary and conclusions in Section 8.

\section{METHODOLOGY}

This study followed a participatory design approach with science teachers [14, 22, 39]. To address its research questions, we conducted three consecutive experiments with science teachers (hereafter referred to as Study 1, 2, and 3).

In Study 1, a group of high-school science teachers who use PeTeL to blend digital learning resources into their teaching was interviewed to understand their perception towards personalization, and how they envision that it can be integrated into their teaching practices. The results of this experiment informed the conceptualization of a group-based personalization strategy, and the design of a mock-up of a learning analytics tool that supports it (enables the teacher to cluster students based on multi-dimensional analysis of past activities, then match an appropriate next-step activity to each cluster).

In Study 2, the mock-up was presented to another group of teachers who participated in a professional development program that focused on co-designing learning analytics tools for PeTeL, in order to learn what type of analytics they would like to receive through the tool, and how they would like these analytics to be explained in a pedagogically meaningful way (e.g., 'explainable learning analytics' [2, 13, 33, 39]).

In Study 3, the results of Study 1 and 2 informed the design of an algorithm that groups students, based on their responses to sets of interactive assessment items selected by the teacher, into clusters of students that exhibit similar response patterns. For each cluster, the rationale behind the clustering is also provided, describing the strengths and weaknesses of each group in terms of the competencies and skills that underlie the items (derived from the item metadata). A controlled experiment evaluated whether providing these analytics to teachers increased their ability to design learning sequences that are more adapted to the needs of individual students.

Below we describe each of these experiments in more detail. 


\section{STUDY 1: TEACHERS' PREFERENCES TOWARDS APPLYING PERSONALIZED INSTRUCTION}

The first step of our research focused on two main issues: i) understanding teacher perception of personalized instruction in terms of its benefit to students and the challenges involved in implementing it in K-12 blended-learning scenarios; and ii) defining with teachers a feasible personalization strategy: how and where personalization can be integrated into their teaching practices.

\subsection{Procedure and Materials}

To study these issues we conducted semi-structured interviews with five high-school science teachers. Interviewees had at least two years of experience teaching in blended learning environments (with PeTeL or other LMSs), and at least five years of experience as science teachers.

The interviews (30-60 min each) focused on the following key questions:

- Do you believe that assigning different learning activities for individual students or groups of students in your class will improve student learning outcomes?

- How and where personalized instruction can be integrated into your teaching practices?

- Do you actually implement such practices in your classroom? If yes, can you describe this in more detail? If not, why not?

\subsection{Analysis and Results}

In terms of their beliefs, all the interviewees expressed a positive view about the potential power of personalization to improve students' learning outcomes. Nevertheless, they stated that they currently prefer to base their pedagogy on a principle that can be described as "one-size that fits most (of the students in the class)". Rare (if any) application of a personalized teaching approach was reported by the teachers, and the main difficulty being mentioned was managing an in-class learning process where every student works on an individual task. As one of the teachers stated: "It [assigning individual tasks to every student] is simply impossible, it will take a huge amount of time, which I do not have", while another stated: "I will be totally out of control in such a scenario".

However, while the interviewees were skeptical about assigning and managing in-class personalized learning activities on the level of the individual student, they were confident that they will be able to monitor and control personalization on the group level - assigning different tasks at the same time to several groups of students. As one teacher explained: "I can move between groups, assign a challenging task to a group of stronger students, and provide more attention to a group of struggling ones". All the teachers mentioned in-class group work as a routine practice, but mentioned that the grouping is rarely done based on cognitive analysis of students' current state of knowledge. As one teacher mentioned: "I use this practice in my classes, but I give them the freedom to choose a group, I do not care. The groups will be given the same tasks", while another stated: "[To divide students into groups] I use my intuition. I know who is strong and who is weak". One teacher mentioned that he usually assigns the students into groups of 'weak' and 'strong' students at the beginning of the school year, and then uses this grouping during the entire year (!).

The consensus among the teachers about their capability of managing group work (whether assigned with similar or different tasks) and their positive views about personalization, suggests that, given proper information and support, teachers can successfully integrate personalization strategies that operate on the group level, without being required to make a substantial change in their teaching practices. 
Drawing on these findings, we decided to focus our attention on developing a learning analytics-based solution that will support group-level personalization, enabling teachers to design group-level task-assignment that is based on multi-dimensional analysis of student responses to auto-graded interactive assessment items. Thus, our next step was designing a mock-up of such a tool and studying what type of analytics teachers would like to receive through it.

\section{STUDY 2: CO-DESIGNING A LEARNING ANALYTICS DASHBOARD FOR PERSONALIZED INSTRUCTION}

Following the results of Study 1, Study 2 focused on designing a mock-up of a dashboard that supports group-level analysis and task assignment. Then, our goal was unpacking teachers' perceptions regarding how to explain the algorithmic decisions that underlie the visualized analytics - student clusters and their response patterns - in a pedagogically meaningful way, making them actionable for assigning follow-up learning sequences that match the strengths and weaknesses that the multi-dimensional analysis reveals.

\subsection{Procedure and Materials}

Dashboard. We created a mock-up of the dashboard to demonstrate its main functionalities and how it will be integrated into PeTeL. The mock-up was designed by a multidisciplinary team consisting of physics and biology science teaching researchers, educational data scientists, UX/UI designers, and teachers. The mock-up visualizes how, based on the analysis of a set of PeTeL online diagnostic activities that are chosen by the teacher (each consisting of a sequence of problem-solving tasks that require mastering several skills and competencies), the algorithm analyzes students' responses and clusters them into groups of students who exhibited similar response patterns. Next, the mock-up demonstrates how the dashboard can be used to assign different follow-up activities to different student groups. The proposed groups are presented as circles containing icons for each student belonging to the group. Student and group characteristics were visualized as information boxes that appear by clicking on the corresponding icons (see Figure 1).

Procedure. The experiment was conducted with a group of twelve high-school science teachers who participated in a year-long professional development program focusing on co-designing learning analytics features for PeTeL. Each of them had at least two years of experience applying blended-learning instruction with PeTeL, and at least five years of experience as a science teacher.

The experiment had two main goals. First, to learn whether teachers perceive the group-based learning analytics strategy and the dashboard that was designed to support it, as effective. Second, given that the grouping is based on decisions made by an algorithm, what type of information they would like to receive on the grouping in order to make its analysis useful for assigning an appropriate follow-up learning sequence for each group of students (namely, we wanted their assistance in addressing an 'explainable learning analytics' dilemma).

The experiment spanned over two hours and was conducted remotely due to Covid-19 constraints. In its beginning, we demonstrated the dashboard to the teachers, explained the approach of group-level personalization that is based on multi-dimensional analysis, and the algorithmic process that clusters the students.

To understand teachers' authentic, unbiased perceptions of what analytics can be beneficial for supporting such personalization strategy, we presented them with blank information boxes (see Figure 1), with no specific information in them, and asked the participants to fill these boxes with the types of analytics that would assist them in creating personalized learning sequences for each group.

In the first step, we asked teachers to fill (individually) an on-line review with two open-ended questions: 


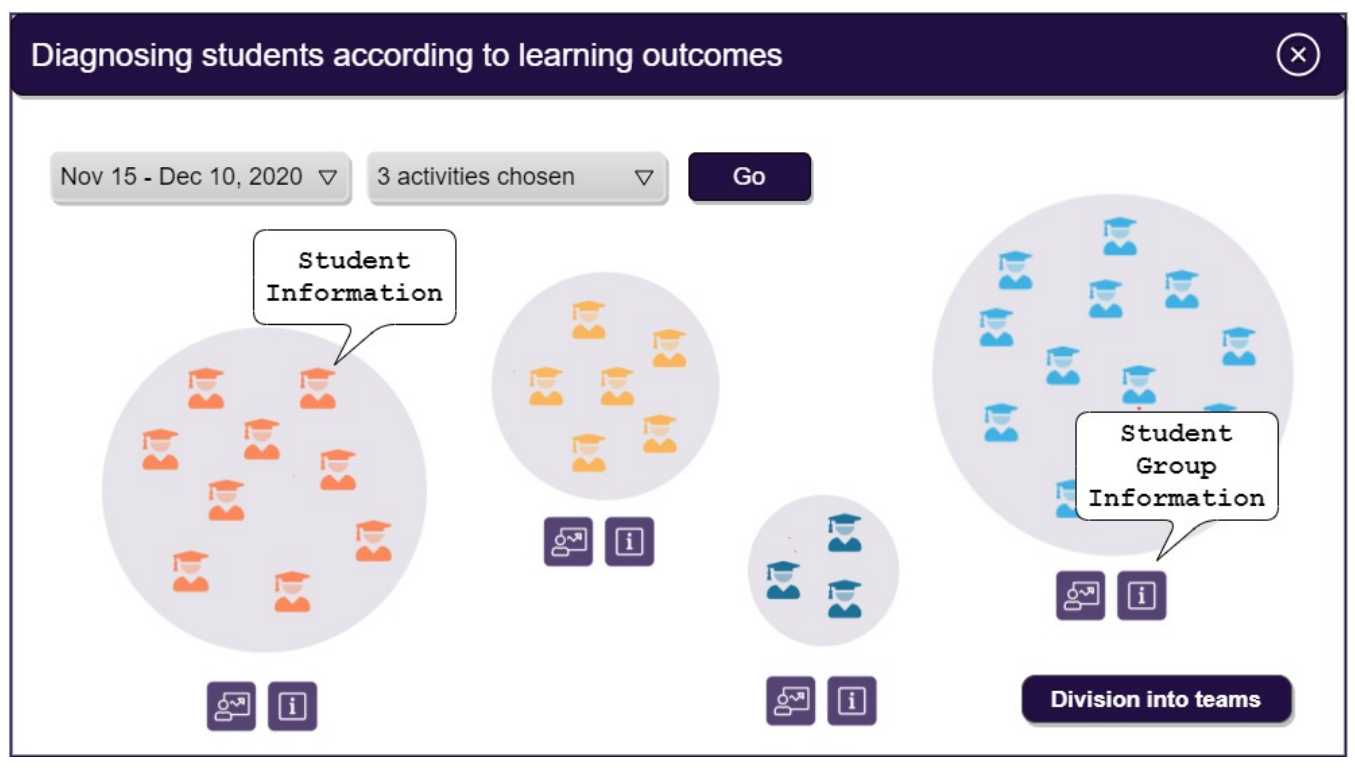

Fig. 1. The mock-up of the clustering dashboard.

(1) Assuming that you have decided to assign an individual task to each student (e.g., for homework) or student group (e.g., in-class group work), what information would you like to receive in the information boxes?

(2) How do you intend to use this information for planning a personalized follow-up activity or learning sequence?

Then, the teachers were divided into four discussion groups. The members of each group were asked to present their ideas to the group and discuss what information they would like to be presented in the information boxes. Since these are 'explanations' that give pedagogical meaning to algorithmic decisions, we refer to them as semantic information. Each discussion group summarized its ideas on a shared sketch (one per group). An example of such a sketch is presented in Figure 2. The discussions were facilitated and monitored by the mock-up design team members.

\subsection{Analysis and Results}

The ideas proposed by the teachers were extracted from teacher survey answers and the four shared sketches used in the group discussions. Their analysis followed the general scheme for quantifying qualitative data [9]. The ideas were analyzed and divided into three categories: i) aggregated performance measures of the students in the group (e.g., average grade per question, average time spent on a task); ii) analysis by disciplinary and crosscutting concepts that experts tagged on the problems in the activities (namely, which skill/competencies students in the group master, and in which they struggle); and iii) between-group analysis that highlights, using the above measures, the ones that discriminate between the groups (e.g., marking that a certain concept, or problem, is high on one group and low in another). For each category and measure, we counted the number of teachers and teacher groups who mentioned it in the review (presented under columns 'Teacher' and 'Group' in Table 1). The ideas proposed by the largest numbers of teachers/groups are highlighted in bold text.

As emerged from the data (see Table 1), the teachers were most interested in information that explains the groups in terms of competencies - both disciplinary and cross-cutting concepts. Interestingly, teachers were interested to know 


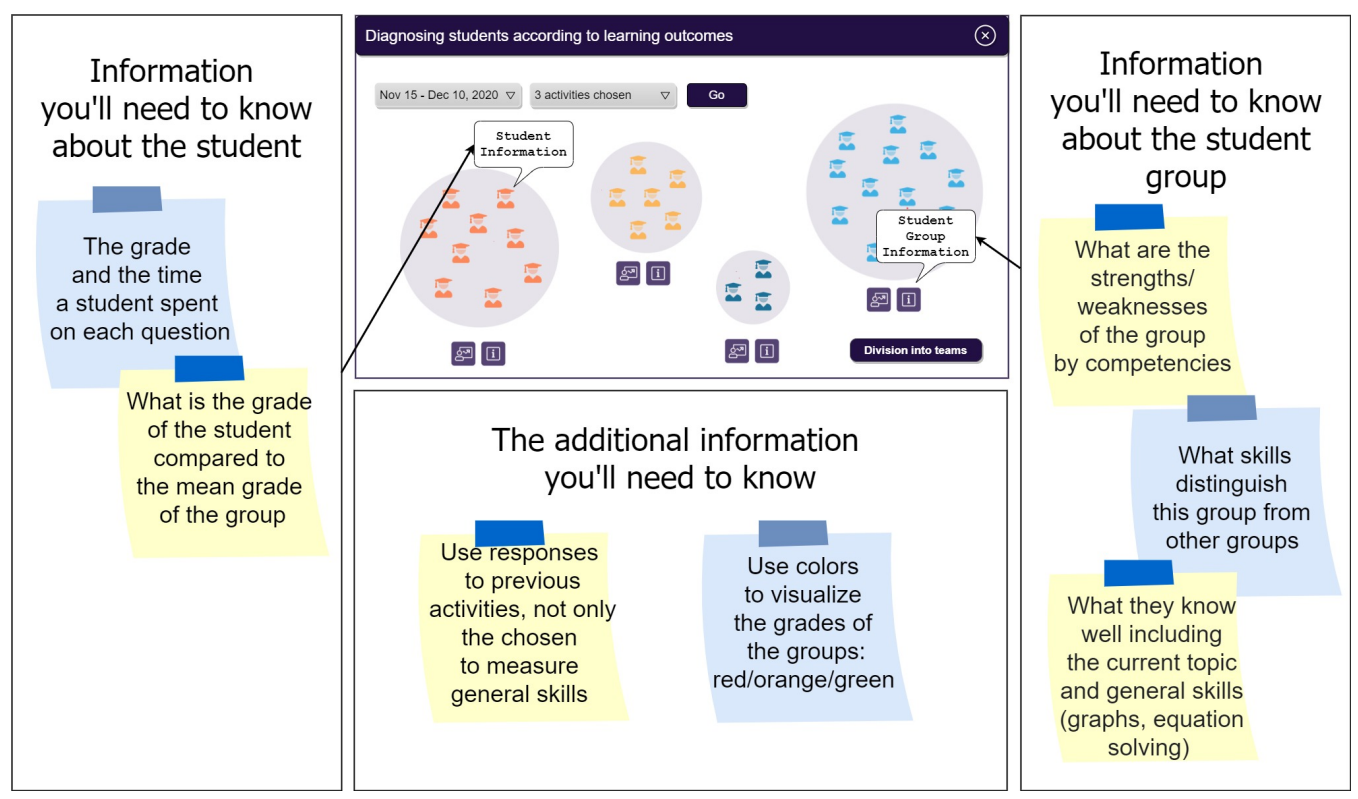

Fig. 2. The resulting sketch produced by one of the discussion groups.

Table 1. Group-level analytics proposed by the teachers.

\begin{tabular}{lll}
\hline Analytics & Teacher & Group \\
\hline (1) Aggregated performance measures & & \\
\hline Mean time on the task & 2 & 2 \\
Mean time per question & 2 & 1 \\
Mean grade on the task & 3 & 1 \\
Mean grade per question & 1 & 0 \\
Number of uncompleted tasks & 3 & 1 \\
\hline (2) Competency-based analysis & & \\
\hline Weak skills/competencies & $\mathbf{6}$ & $\mathbf{4}$ \\
Strong skills/competencies & $\mathbf{6}$ & $\mathbf{3}$ \\
Knowledge levels in sub-topics & $\mathbf{4}$ & $\mathbf{3}$ \\
Categories of common errors/misconceptions & 1 & 2 \\
Missing background skills/conceptual knowledge & 2 & 1 \\
\hline (3) Between-groups analysis & & \\
\hline Differences between the groups & 2 & 2 \\
Similarities between the groups & 2 & 1 \\
Distinguishing parameter/s of a group & 1 & 0 \\
\hline
\end{tabular}

not only in which concepts each group struggled, but also in which concepts it performed well. The teachers identified such analytics as very useful for two use-cases that 'call' for group-based personalization: one is in-class group work, and the other being homework assignments that are adapted on the student- or group-level.

This co-design process around the mock-up and the use-cases also highlighted that teachers want the ability to integrate their local knowledge into the system-provided analytics. We identified three types of information that they 
wanted to add. First, augmenting the algorithmic decision with non-cognitive information, such as social relations between the students, which are not considered by the algorithm, but may affect collaborative work (in case that this was the teacher's plan for the next class activity). Second, since in practical scenarios, data on some of the students is typically missing (e.g., if they did not complete the activity), they wanted the ability to fill the gap either by imputing data or by mimicking the algorithmic decisions for such students. Third, the teachers wanted the ability to override any decision made by the algorithm because they 'know their students better'. (To address these requirements, the eventual design of the tool includes features that give teachers 'super-power' to override the algorithm decisions. See Section 6.)

Based on these findings, the next step focused on evaluating whether explaining the clustering in terms of competencies actually enhances teachers' ability to design and assign follow-up activities that address the specific needs of each group. To study this, we evaluated in a controlled experiment this personalization strategy, which combines algorithmic-based grouping with competency-based explanations. This experiment is described in the next section.

\section{STUDY 3: EVALUATE THE 'ALGORITHMIC-BASED GROUPING WITH COMPETENCY-BASED EXPLANATIONS' PERSONALIZATION STRATEGY}

Following the results of Studies 1 and 2, we conducted an intervention study with two main goals. The first was developing an algorithm for automatically dividing students into groups based on their responses to the interactive assessment. The second was evaluating whether the proposed strategy of performance-based clusters that are explained in terms of expert knowledge - the skills and competencies that underlie the problems according to which the students were clustered - enhances teachers' ability to tailor learning sequences that match student needs.

\subsection{Procedure and Setting}

The experiment was conducted during a workshop as part of a thirty-hour professional development program for high school Biology teachers $(n=48)$. In the experiment, teachers were presented with the following realistic classroom scenario: "After administering the Gazelle interactive activity (see below) to your students, you wish to divide the class into groups of students with similar levels of competencies, and assign to each group (or students in that group) a follow-up learning sequence that is most appropriate for them."

The teachers were randomly divided into experimental and control groups of roughly equal sizes. The teachers in both groups received the results of a synthetic class composed of actual student data (data is described below), clustered according to their performance on the interactive activity (the clustering algorithm is provided in Appendix A). The main difference was that in the treatment group the clusters were explained with expert knowledge, while in the control group no such semantic information on the clusters was provided. As defined above, the task was to define a personalized follow-up learning sequence for the students in each group. The teachers received fifteen minutes for completing this task. (In a different questionnaire, not presented here, the teachers were requested to estimate the time it would take them to design adaptive follow-up learning sequences; more than $90 \%$ of them estimated that it would take at least fifteen minutes). Below we describe the Gazelle learning activity, the expert knowledge, and the clustering procedure.

\subsection{The Gazelle Learning Activity.}

The data that was presented to the teachers was taken from an interactive activity administered to students through PeTeL. The activity aims to develop and practice inquiry skills through analyzing an authentic database on Gazelles, including data on 1175 gazelles (hence referred to as the 'Gazelle Activity'). The activity included ten items that focus 
on three competencies: inquiry skills, data literacy, and critical thinking and reasoning. These competencies are defined by the Israeli Ministry of Education as core components of the high-school biology curriculum. Inquiry skills, in this context, include defining research questions, identifying variables, using controls, and understanding the importance of repetitions. Data literacy includes, in the Gazelle activity, creating and analyzing graphs. Critical thinking and reasoning refer to issues such as deriving conclusions and critically assessing them during the activity (further description of the activity is provided in Appendix B). While some questions in the activity focus on a single competency, others combine several, and answering the last task, in which the students are requested to perform independent research, demands mastering all three competencies. The mapping of the items to competencies was done through expert task analysis and was validated by 36 experienced biology teachers. Table 2 summarizes the different competencies practiced in each question of the activity (denoted Q Matrix [10,36]).

Table 2. Q Matrix for the Gazelle activity

\begin{tabular}{|c|c|c|c|c|c|c|c|c|c|c|}
\hline \multirow[t]{2}{*}{ Competencies } & \multicolumn{10}{|c|}{ Questions } \\
\hline & 1 & 2 & 3 & 4 & 5 & 6 & 7 & 8 & 9 & 10 \\
\hline Inquiry Skills & + & & & & & + & & & & + \\
\hline Data Literacy & & + & + & + & + & & + & & & + \\
\hline Critical Thinking and Reasoning & & & & + & + & & & + & + & + \\
\hline
\end{tabular}

The task analysis and the item-to-competencies mapping that it yielded, represented in the Q Matrix, reflect expert knowledge and provide semantic information on the items. This enables to analyze student performance on the instrument according to the competencies that underlie its items, providing a deeper understanding of students' strengths and weaknesses.

\subsection{Constructing a synthetic class from real student data}

The Gazelle activity was administered to 100 students through PeTeL, yielding a $100 \times 10$ response matrix. A clustering algorithm (described in the Appendix A) divided the students into four groups. One of the resulting clusters consisted of students who completed successfully the entire activity, and as such was not interesting for further analysis of student difficulties. We focused on the three remaining clusters. For these clusters, the algorithm provided the items that distinguish each cluster. Two experts analyzed the competencies that underlie these items (the $Q$ Matrix that is presented in Table 2), yielding competency-based explanations that provide semantic information on the clusters. (We note that although the explanations were formulated by experts, this explanation-building phase can be constructed automatically from the $\mathrm{Q}$ Matrix and the items that the clustering algorithm identifies as most distinguishing between the groups.) Once these clusters and the explanations were defined, we sampled $5-6$ students from each of the three clusters to form a synthetic class of 16 students.

\subsection{Task and the experimental and control conditions}

As mentioned above, the teachers' task was to examine students' performance and define follow-up learning sequences. As a baseline, the teachers in both groups (experimental and control) received an analytics dashboard (a sketch of) that presents the students in each cluster and their grades, and cluster-level information - which questions were easy/difficult for students in each cluster (we refer to this as 'Analysis by Questions'). This is presented in Figure 3 as 'Control'. The experimental group received, in addition, cluster-level information that is based on expert knowledge - 
the competencies mastered/not mastered by the group members. These analytics are based on analyzing the Q Matrix of the items that each cluster mastered or not, and thus provide a semantic, expert-based description of the clusters. They appears as the right column in Figure 3, as 'Experimental'.

Both groups also received the raw data used to compute the analytics in the dashboard: the mapping of questions to competencies (Q Matrix), and the student response matrix. This means that teachers in the control group could, theoretically, extract the semantic description of the clusters by combining the information in the Q Matrix and the Response Matrix.

With this information, all teachers received the same (individual) task: Define three follow-up learning sequences, one for each group of students.

\section{Diagnosis students according to learning outcomes}

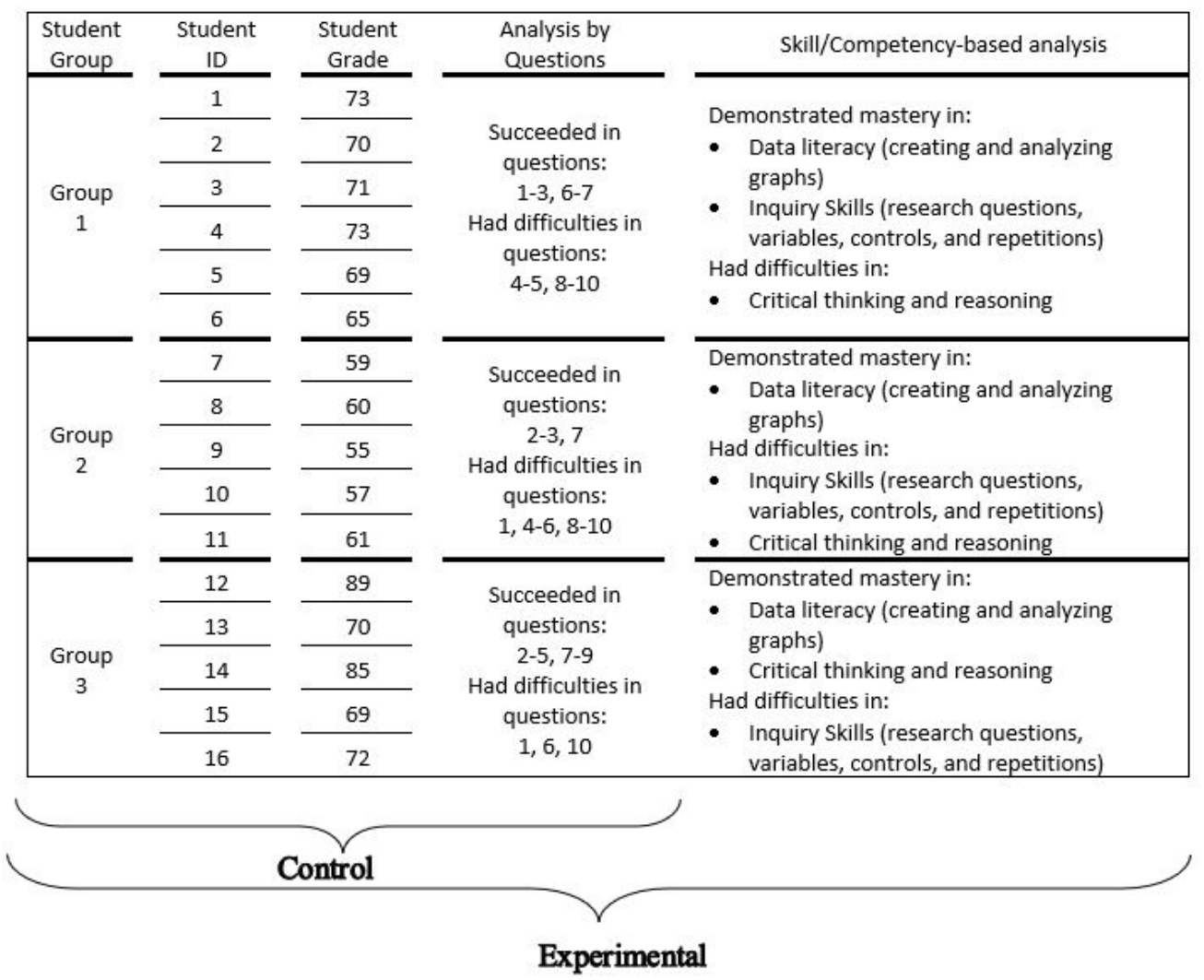

Fig. 3. Dashboard sketches for the Control and Experimental groups.

\subsection{Analysis and Results}

28 teachers were included in the Experimental group and 20 were included in the control group. In total $48 \times 3=144$ follow-up learning sequences were collected. 
5.5.1 Evaluating teacher-designed learning sequences. To evaluate teacher performance on the task we graded the follow-up learning sequence proposed by each teacher for each student group. The grading was intended to capture how well the proposed treatment addressed the specific needs of the students in the group. The grading included the following scale:

- 0 - no learning sequence was proposed

- 1 - the proposed learning sequence does not address the needs of the group

- 2 - the proposed learning sequence is partial or too coarse

- 3 - the proposed learning sequence is specific to the needs of the group

The grading of teacher sequences according to this 0-3 scale was done by a domain expert (the second author of the paper) in a blinded fashion, to avoid possible biases. That is, when grading, the expert did not know to which group experimental or control - each analyzed sequence belonged.

Next, we compared the grading between the groups to evaluate the effect. Figure 4 presents the distribution of grades that teachers' proposals for each group received. As demonstrated in the figure, learning sequences designed by the teachers in the experimental group tended to receive higher grades, meaning that the expert found them as more appropriate for the groups' needs. To check if there is a statistically significant difference between the control and experimental groups, we used a non-parametric Wilcoxon sum rank test as the distributions were not normally distributed ( $\mathrm{p}$-value $<0.05$ in the Shapiro-Wilk test). The Wilcoxon test $\mathrm{p}$-value was 0.018 , indicating that the grades given to the sequences designed by the teachers in the experimental group were significantly higher than the grades given to sequences designed by the teachers in the control group (using a 95\% confidence level). Based on these results, we concluded that providing competency-based semantic information on student clusters has a statistically significant positive effect on teachers' ability to create personalized follow-up learning sequences.

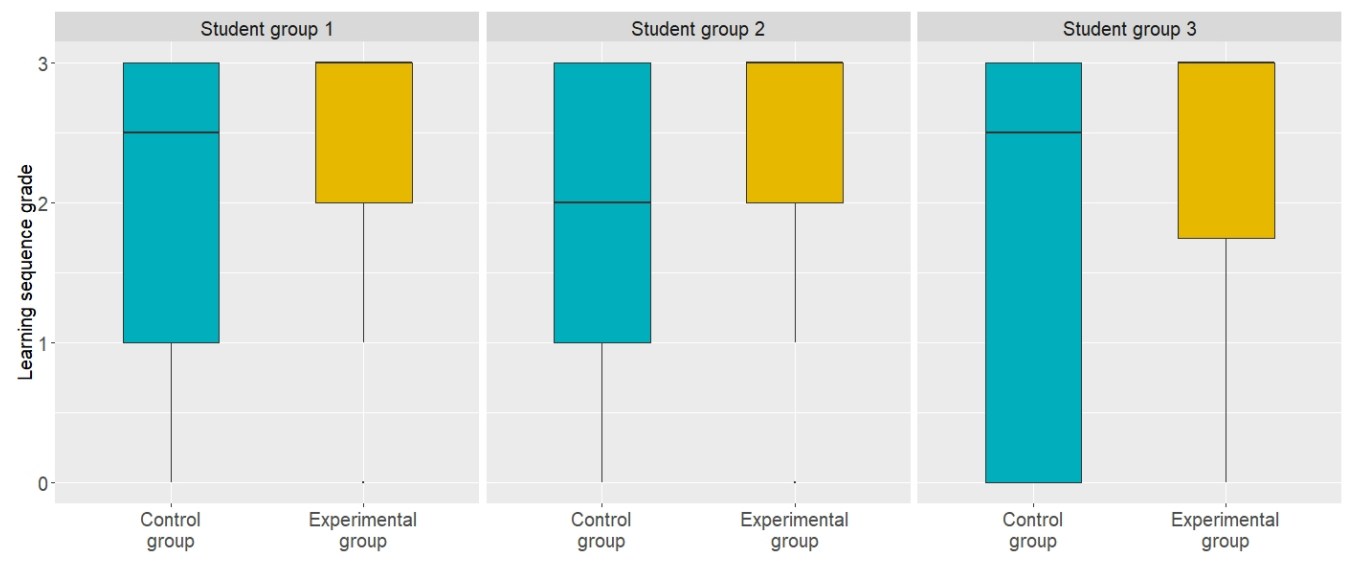

Fig. 4. Grades given to personalized learning sequences designed by the teachers for each group of students. The distribution of the grades of the teachers in the control group is colored in blue and the distribution of the grades of the teachers in the experimental group is colored in yellow. 


\section{TOOL: DESIGN \& IMPLEMENTATION}

Based on the results of Studies 1-3, we developed a learning analytics tool that implements this 'algorithmic-based clustering with competency-based explanations' approach and integrated it into PeTeL, a free online learning platform that is in use by more than 1000 science teachers. In the current and next school year, we intend to monitor its use and conduct additional experiments in order to evaluate its efficacy and iteratively improve it. A screenshot of the teacher dashboard with an explanation of its different components is presented in Figure 5.

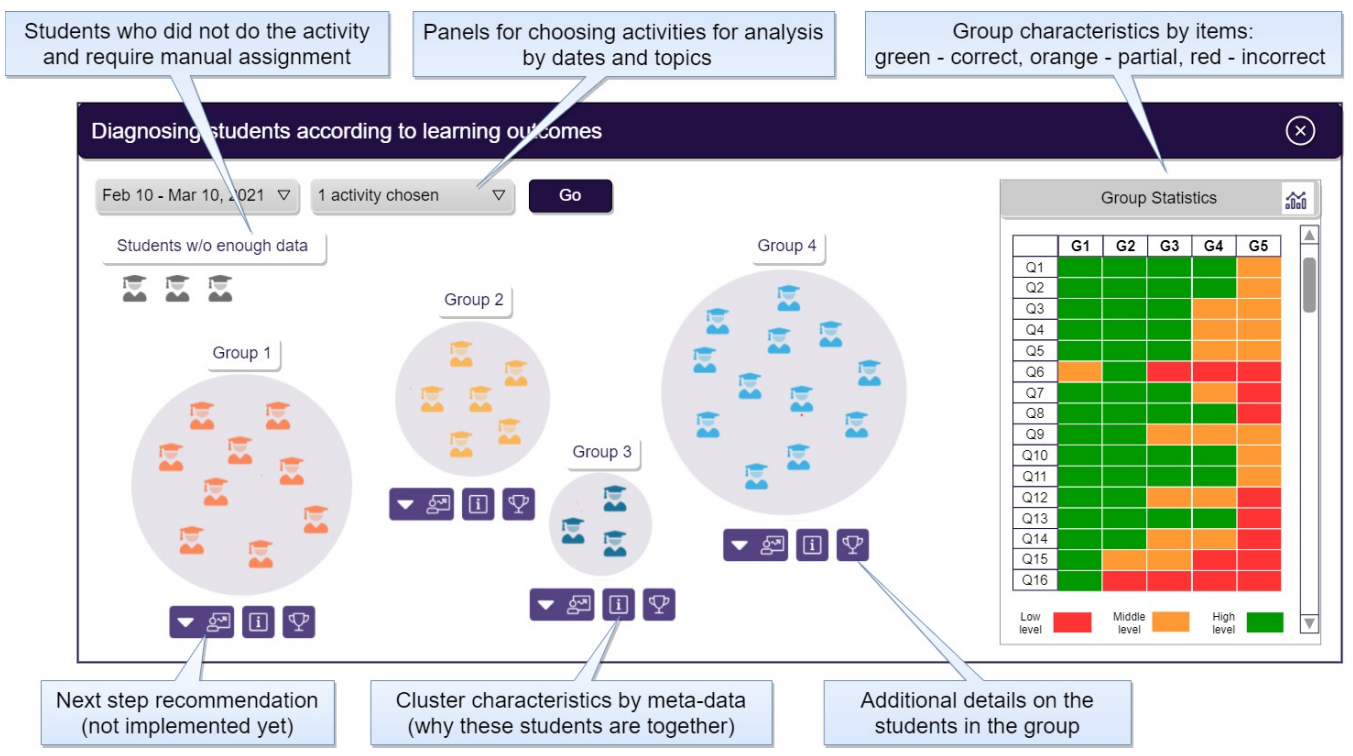

Fig. 5. The dashboard of the tool (translated from Hebrew). The tool presents clusters of students and explains the key characteristics of each group in two ways: metadata (e.g., concepts/skills, under 'i'), and items (right table). To address class constraints and incorporate teachers' knowledge of the students, the tool supports overriding the algorithm's decision by dragging students between groups, as well as assigning ones on which there is not enough information (e.g., did not do the activity). The tool includes a button for recommendation on activity for the group, which is under development.

\section{DISCUSSION AND CONCLUSIONS}

We have presented a case study of a co-design process with science teachers aimed at designing a learning analytics tool that will assist them in providing personalized instruction in K-12 science classrooms. Our design process consisted of three consecutive experiments designed to i) shed light on where and how science teachers believe that they can integrate personalization into their teaching practices; ii) reveal what types of analytics teachers believe would assist them in doing so; and iii) study if providing these analytics actually increases teachers' ability to tailor their instruction to the needs of students with different types of difficulties.

The results of Study 1 indicated that teachers are interested in providing more personalized instruction, but find it infeasible to implement in-class personalized instruction on the level of the individual student. However, they were interested in solutions for group-level personalization, as group work is more manageable and already part of their teaching practices. While grouping can be based on various factors, they focused on cognitive-based ones - nuanced content knowledge and cross-cutting skills ('micro-level classroom analytics' [41]). In fact, the teachers explicitly 
identified this as a gap - while they would like to divide students into groups based on their current knowledge state, and assign to each group a learning sequence that is most relevant to that state, they typically base the grouping on intuition and/or assign the same sequence to all the groups, due to the absence of real-time analytics.

Based on these findings, we decided to focus our attention on developing a learning analytics tool that would enable teachers to manage group-level learning sequence-assignment that is based on multi-dimensional analysis of student responses to auto-graded interactive assessment instrument/s. Thus, our next step was designing a mock-up of such a tool, and studying what type of analytics teachers would like to receive through it and how its use fits into their teaching routines.

As described in Section 4, the teachers were most interested in semantic information that describes the groups in terms of competencies and skills - both disciplinary and cross-cutting ones (e.g., reading from graph) - so they could plan and search for a tailored intervention. On the contrary, teachers show low interest in information that is less relevant for planing an adaptive intervention, such as comparisons between the groups, or aggregated analysis that is not very 'actionable', such as total grade on the task.

In addition, Study 2 highlighted that teachers want the ability to integrate their local knowledge into the systemprovided analytics. We addressed three types of incentives for doing so: i) augmenting: considering types of information that are not available to the algorithm, such as social relations between the students; ii) imputation: filling the gap for students on which the algorithm does not have enough data; and iii) override: alter the algorithmic decision when it is perceived as making an inaccurate judgment on some of the students.

In order to apply learning analytics to real-world educational settings, instructors need to translate system-provided information into locally meaningful knowledge [20, 41]. Our findings demonstrate that such translation may inherently require filling information gaps and provide a more nuanced understanding of the types of gaps that K-12 science teachers want to fill in order to apply analytics for decision making in their classroom.

As we demonstrated in the controlled experiment reported in Study 3, providing competency-based semantic information on student clusters has a statistically significant positive effect on teachers' ability to create tailored follow-up plans that address students' current state of knowledge, namely, providing personalized learning sequences.

Using clustering techniques for classifying students into profiles in order to inform adaptive intervention is a general learning analytics technique $[24,30]$. The novelty of our study lies in its holistic approach for addressing an authentic, under-explored challenge: Supporting teachers in planning personalized classroom activities, using 'explainable learning analytics' that are both pedagogically meaningful for teachers and can be automatically derived from item-level metadata (represented as a Q Matrix). The effectiveness of this approach was demonstrated through a controlled experiment and led to the development of a learning analytics tool that is now available on a free learning platform that is in use by more than 1000 high-school science teachers. Since demonstrations of successful end-to-end co-design processes of learning analytics tools, especially for K-12 contexts, remain rare in the literature [14], we believe that both the learning analytics tool that we propose, and the co-design process that produced it, are a valuable contribution to the learning analytics literature. In addition, our findings shed light on the information gaps between system-provided analytics and class reality, either objective or subjective, that teachers need to fill in order to make learning analytics meaningful for their local context.

\subsection{Limitations}

The main limitation of our research is its sample. It was conducted with a few groups of physics and biology high-school teachers who use PeTeL and chose to participate in professional development programs at Weizmann Institute. These 
teachers may not be a representative sample of the entire teacher population. Another main limitation is the context. Study 3 evaluated the efficacy of the proposed personalization approach on a single activity in Biology. While the results were statistically significant, establishing external validity of this approach to different activities and different groups of teachers requires further experiments. However, this weakness is the result of the holistic viewpoint and the end-to-end nature of our research that attempts at understanding the system as a whole, at the cost of being more context-dependent. In fact, taking a 'holistic' perspective, rather than a 'reductionist' one, is one of the key characteristics of learning analytics research [34].

\subsection{Future Research}

This study has several directions for future research. Among the interesting ones are questions concerning 'explainable learning analytics', and in particular, how to automatically render explanations that are pedagogically meaningful for teachers.

\section{SUMMARY}

This paper presents the results of a participatory design study aimed at co-designing with science teachers a learning analytics tool that will assist them in implementing a personalized pedagogy in blended learning contexts. The study included three experiments, focusing on where and how science teachers believe that they can integrate personalization into their teaching practices, what types of analytics would assist them in doing so, and whether providing them with these analytics actually increases their ability to design learning sequences that better fit students weaknesses and strengths with respect to core scientific skills.

The main contribution of this research is twofold. First, it presents a practical end-to-end personalization approach that combines algorithms, data, and expert knowledge to assist teachers in providing instruction that fits individual students' needs and fits the reality of blended learning in the science classroom. Its analytical core is based on a real-time clustering algorithm and an explainable-AI scheme that can automatically build pedagogically meaningful explanations from item-level meta-data (Q Matrix). Second, it demonstrated how such a holistic learning analytics solution can be built through a participatory design process. This process also highlighted the types of knowledge teachers want to add to system-provided analytics in order to make them relevant to their local context, and how this requirement can be met by giving them 'super power' to override algorithmic decisions. As a practical contribution to teachers and learners, the results of this research informed the design of a new learning analytics tool that was integrated into a free online learning platform that is being used by more than 1000 science teachers.

\section{ACKNOWLEDGMENTS}

TN is grateful to the Azrieli Foundation for the award of an Azrieli Fellowship.

\section{REFERENCES}

[1] Giora Alexandron, José A Ruipérez-Valiente, Zhongzhou Chen, Pedro J Muñoz-Merino, and David E Pritchard. 2017. Copying@ Scale: Using harvesting accounts for collecting correct answers in a MOOC. Computers \& Education 108 (2017), 96-114.

[2] José M Alonso and Gabriella Casalino. 2019. Explainable artificial intelligence for human-centric data analysis in virtual learning environments. In International Workshop on Higher Education Learning Methodologies and Technologies Online. Springer, 125-138.

[3] Pengcheng An, Kenneth Holstein, Bernice d'Anjou, Berry Eggen, and Saskia Bakker. 2020. The TA Framework: Designing Real-time Teaching Augmentation for K-12 Classrooms. In Proceedings of the 2020 CHI Conference on Human Factors in Computing Systems. 1-17.

[4] Karen T Arnesen, Charles R Graham, Cecil R Short, and Douglas Archibald. 2019. Experiences with personalized learning in a blended teaching course for preservice teachers. Journal of Online Learning Research 5, 3 (2019), 275-310. 
[5] Ryan Baker, Jason Walonoski, Neil Heffernan, Ido Roll, Albert Corbett, and Kenneth Koedinger. 2008. Why students engage in "gaming the system" behavior in interactive learning environments. Journal of Interactive Learning Research 19, 2 (2008), 185-224.

[6] Ryan S Baker. 2016. Stupid tutoring systems, intelligent humans. International fournal of Artificial Intelligence in Education 26, 2 (2016), 600-614.

[7] Benjamin S Bloom. 1984. The 2 sigma problem: The search for methods of group instruction as effective as one-to-one tutoring. Educational researcher 13, 6 (1984), 4-16.

[8] Hilda Borko, Sarah A. Roberts, and Richard Shavelson. 2008. Teachers' Decision Making: from Alan f. Bishop to Today. Springer US, Boston, MA, 37-67.

[9] Michelene T. H. Chi. 1997. Quantifying qualitative analyses of verbal data: A practical guide. The fournal of the Learning Sciences 6, 3 (1997), 271-315.

[10] Michel Desmarais et al. 2011. Conditions for effectively deriving a q-matrix from data with non-negative matrix factorization. In Proceedings of the 4th International Conference on educational Data Mining. 41-50.

[11] Pierre Dillenbourg and Patrick Jermann. 2010. Technology for classroom orchestration. In New Science of Learning. Springer, 525-552.

[12] Mollie Dollinger, Danny Liu, Natasha Arthars, and Jason Lodge. 2019. Working together in learning analytics towards the co-creation of value. fournal of Learning Analytics 6, 2 (2019), 10-26.

[13] David Gunning, Mark Stefik, Jaesik Choi, Timothy Miller, Simone Stumpf, and Guang-Zhong Yang. 2019. XAI-Explainable artificial intelligence. Science Robotics 4, 37 (2019).

[14] Kenneth Holstein, Bruce M McLaren, and Vincent Aleven. 2019. Co-designing a real-time classroom orchestration tool to support teacher-AI complementarity. Journal of Learning Analytics 6, 2 (2019), 27-52.

[15] Michael B Horn and Heather Staker. 2011. The rise of K-12 blended learning. Innosight Institute 5 (2011), 1-17.

[16] Sarah K Howard. 2013. Risk-aversion: Understanding teachers' resistance to technology integration. Technology, Pedagogy and Education 22, 3 (2013), 357-372.

[17] Sarah Katherine Howard and Adrian Mozejko. 2015. Teachers: technology, change and resistance. Teaching and Digital Technologies: Big Issues and Critical Questions (2015), 307-317.

[18] Anil K Jain. 2010. Data clustering: 50 years beyond K-means. Pattern Recognition Letters 31, 8 (2010), 651-666.

[19] John King. 2017. Reimagining the role of technology in education: 2017 national education technology plan update.

[20] Kirsty Kitto, Simon Buckingham Shum, and Andrew Gibson. 2018. Embracing imperfection in learning analytics. In Proceedings of the 8th International Conference on Learning Analytics and Knowledge. 451-460.

[21] Roberto Martinez-Maldonado, Andrew Clayphan, Kalina Yacef, and Judy Kay. 2014. MTFeedback: providing notifications to enhance teacher awareness of small group work in the classroom. IEEE Transactions on Learning Technologies 8, 2 (2014), 187-200.

[22] Roberto Martinez-Maldonado, Abelardo Pardo, Negin Mirriahi, Kalina Yacef, Judy Kay, and Andrew Clayphan. 2015. LATUX: An Iterative Workflow for Designing, Validating, and Deploying Learning Analytics Visualizations. Journal of Learning Analytics 2, 3 (2015), 9-39.

[23] Manolis Mavrikis, Sergio Gutierrez-Santos, Eirini Geraniou, R Noss, and A Poulovassilis. 2013. Iterative context engineering to inform the design of intelligent exploratory learning environments for the classroom. Handbook of design in educational technology (2013), 80-92.

[24] Agathe Merceron and Kalina Yacef. 2004. Clustering Students to help Evaluate Learning. In Technology Enhanced Learning, Jean-Pierre Courtiat, Costas Davarakis, and Thierry Villemur (Eds.). Kluwer Academic Publishers, Toulouse, France, 31-42.

[25] Tanya Nazaretsky, Sara Hershkovitz, and Giora Alexandron. 2019. Kappa Learning: A New Item-Similarity Method for Clustering Educational Items from Response Data.. In Proceedings of the 12th International Conference on Educational Data Mining. 129-138.

[26] Luis P Prieto, Martina Holenko Dlab, Israel Gutiérrez, Mahmoud Abdulwahed, and Walid Balid. 2011. Orchestrating technology enhanced learning: a literature review and a conceptual framework. International fournal of Technology Enhanced Learning 3, 6 (2011), 583-598.

[27] Luis P Prieto, Kshitij Sharma, and Pierre Dillenbourg. 2015. Studying teacher orchestration load in technology-enhanced classrooms. In Design for Teaching and Learning in a Networked World. Springer, 268-281.

[28] Carlos G Prieto-Alvarez, Roberto Martinez-Maldonado, and Theresa Anderson. 2018. Co-designing learning analytics tools with learners. 93-110.

[29] Ramkumar Rajendran, Sridhar Iyer, and Sahana Murthy. 2018. Personalized affective feedback to address students' frustration in ITS. IEEE Transactions on Learning Technologies 12, 1 (2018), 87-97.

[30] Cristóbal Romero and Sebastián Ventura. 2010. Educational data mining: a review of the state of the art. IEEE Transactions on Systems, Man, and Cybernetics, Part C (Applications and Reviews) 40, 6 (2010), 601-618.

[31] Cristobal Romero and Sebastian Ventura. 2013. Data mining in education. Wiley Interdisciplinary Reviews: Data Mining and Knowledge Discovery 3, 1 (2013), 12-27.

[32] Andreas Schleicher. 2012. Preparing teachers and developing school leaders for the 21st century: Lessons from around the world. OECD Publishing.

[33] Neil Selwyn. 2019. What's the problem with learning analytics? fournal of Learning Analytics 6, 3 (2019), 11-19.

[34] George Siemens and Ryan SJ d Baker. 2012. Learning analytics and educational data mining: towards communication and collaboration. In Proceedings of the 2nd International Conference on Learning Analytics and Knowledge. 252-254.

[35] James P Spillane. 2012. Data in practice: Conceptualizing the data-based decision-making phenomena. American fournal of Education 118, 2 (2012), $113-141$.

[36] Kikumi K Tatsuoka. 1983. Rule space: An approach for dealing with misconceptions based on item response theory. Fournal of Educational Measurement 20, 4 (1983), 345-354. 
[37] Robert Tibshirani, Guenther Walther, and Trevor Hastie. 2001. Estimating the number of clusters in a data set via the gap statistic. Journal of the Royal Statistical Society: Series B (Statistical Methodology) 63, 2 (2001), 411-423.

[38] Mark Van Harmelen and David Workman. 2012. Analytics for learning and teaching. CETIS Analytics Series 1, 3 (2012), 1-40.

[39] Katrien Verbert, Xavier Ochoa, Robin De Croon, Raphael A Dourado, and Tinne De Laet. 2020. Learning analytics dashboards: the past, the present and the future. In Proceedings of the 10th International Conference on Learning Analytics and Knowledge. 35-40.

[40] Candace A Walkington. 2013. Using adaptive learning technologies to personalize instruction to student interests: The impact of relevant contexts on performance and learning outcomes. Fournal of Educational Psychology 105, 4 (2013), 932-945.

[41] Alyssa Friend Wise and Yeonji Jung. 2019. Teaching with analytics: Towards a situated model of instructional decision-making. Fournal of Learning Analytics 6, 2 (2019), 53-69.

[42] Chuankai Zhang, Yanzun Huang, Jingyu Wang, Dongyang Lu, Weiqi Fang, John Stamper, Stephen Fancsali, Kenneth Holstein, and Vincent Aleven 2019. Early Detection of Wheel Spinning: Comparison across Tutors, Models, Features, and Operationalizations. International Educational Data Mining Society (2019).

\section{A THE CLUSTERING ALGORITHM}

Our clustering method is based on the assumption that students tend to have similar performance on items that require the same skill and competencies [25] and is aimed to divide them into homogeneous groups (clusters) based on their responses to interactive assessment activities. When multiple attempts are allowed we use the first attempt, to reduce the possible effect of guessing or gaming the system [1,5]. The clustering is done on the entire set of students who completed the activity, and each teacher is provided with the projection of the clustering to her class. Basing the clustering on the entire dataset, rather than on the data of each teacher's class, increases the robustness and stability of the clusters and enables more nuanced grouping. The clustering is done using K-means Clustering [18], and the number of clusters is determined with Gap Statistics [37].

To create the automatic descriptions for the clusters, we calculate per cluster the average success rate (over the cluster members) for each one of the 10 items. An item is considered as 'correct' (incorrect) for a cluster if at least $80 \%$ of the students in the cluster solved the item correctly (incorrectly). The $80 \%$ threshold was chosen judgmentally with the pilot teachers.

\section{B THE GAZELLE ACTIVITY}

The gazelle data-driven activity focuses on practicing inquiry and data literacy in an authentic context for high school biology students. It starts with a short introduction to the database content and explains how the data were gathered. The students are presented with a database of 1175 cases of gazelles collected from 1947 until to date. In order to introduce the students to databases and the used platform, the activity begins by studying a given (simple) research question, and the students are asked to define the variables of this question. Later, the students are instructed to data visualization and are asked about the characteristics of the data points in the graph. This aims to connect the specific data points from the graph to the broader data presented in the database. In the next task, the students add the least square line to the graph and note that the data do not perfectly fit the trend line, but include variability of the data points. The variations in characteristics among individuals of the same species call for introducing biological content knowledge when analyzing and interpreting the data. Next, the students are asked to create a graph of the weight of male vs. female gazelles, calculate the mean weights, compare the data they extract from the database to information from Wikipedia and evaluate the credibility of the different data sources. In the last part of the activity, the students are asked to select a research question that interests them and to investigate it independently: after practicing visualizations and analyzing different graphs, they are equipped to ask original research questions and answer them through the gazelle database. This final section integrates both inquiry skills and data literacy, together with critical thinking. 